\title{
Pedagogy Integrated with Information Technology to Promote Its Effectiveness Should be Keen on Humanities
}

\author{
Sophia Ming Lee Wen* \\ College of Humanities and Education, Taiwan Shoufu University, Taiwan \\ *Corresponding author: Sophia Ming Lee Wen, College of Humanities and Education, Taiwan Shoufu University, Taiwan \\ Submission: 僵JAugust 21, 2017; Published: 㭗 September 25, 2017
}

\section{Opinion}

The model of integrating technology into teaching and learning has become a hot trend to promote the effectiveness in such a ubiquitous era. Some might excuse for the limited function and shortage of computing and technology information, including its system, platform and skills users need. However, a success and effectiveness of teaching, in terms of taking care of any students' needs and success, including their background knowledge, learning interests, styles, learning achievements and so on should be considered in all, and furthermore, teaching and learning difficulties should be solved.

There are numbers of interesting issues emerge from the movement of learner-centered educational theory by which no child should let behind and information technology therefore is taken as an effective tool for teachers to help every students reaching their own learning objectives. What, for instance, the nature of pedagogy is, and the virtual relationship between teachers and students displaces traditional instruction via textbooks and faceto face modes of learning. In order to establish a balanced and yet critical theory of the nature of e-learning, research articles concerning theoretical and empirical perspectives for the effective use of technology tools in teaching and learning are all needed to explored a step further to identify the cultural consensus and to meet with the nature of education, viz. subjectivity with free will to be involved in the process of teaching and learning.

Pedagogy and technology is not restricted to electronic educational technology, but is broadly related to its reflection on the metacognition, in terms of instrumental rationality. Therefore, any researches on information and communication technology, computer-based and/or on-line teaching and learning, e.g. flipped classroom, MOOCs etc., innovative teaching and learning, such as professional development community, collaboration teaching and learning constitute of the scope of philosophy of pedagogy and its reflection through e-learning, line or face-book etc.

In other words, what is the relationship between technology and human being? What should education concern more than people those who provoke their ideas from either politic, economic or information technology? That is, an ethical reflection on the behaviors of teachers and students to modeling an effective educational dialogue with excellent quality, in terms of education with free will and computer control is fundamentally significant for both teachers and learners.

It is in this way, the argument about who can control the world in such a technology era could be discussed and the question about the nature of education will be clarified thoroughly as much as a consensus between traditional culture and website internet ethics may be reached through the following topics:
a. Is human behavior human or inhuman in such an internet era today? And why?
b. Who should take responsibilities for life flourishing when human beings have become alien from each other because almost all have been involved in the virtual world via internet technology?

c. What ethic must be facilitated with the booming of website cloud technology?

After acknowledging the consciousness of a subject, playing as an agent in such a ubiquitous era, teachers should acknowledge that the degree of success teachers having in using technology for instruction depends upon their ability to keep students away from an instrument of traditional format of learning. Similarly, students should be able to perceive their own subjectivity, free will, to refuse any indoctrination. In contrast, teachers and students are aware of their rights to make decisions to learn or no to learn, and whether or not they want to learn.

That is, the enlightenment of an autonomous learning will provide both teachers and learners a chance to widen the perspectives to implement technology into the classroom more successfully, in terms of humanities. It is in this idea that students will be encouraged to engage themselves in developing learning objectives, selecting methods of learning, and arranging feedback, 
evaluation and assessment strategies in pedagogy. Therefore, information technology in pedagogy is commonly defined as a technical device or tool used to enhance instruction, rather than a unique criterion for a successful teaching and learning.

It is important that teachers use a variety of teaching methods, and students are getting use to engage in learning, sharing and creating knowledge. On the other hand, with the application of various technologies into pedagogy, teachers with humanities should not isolate their teaching from information technology, but take into consideration the pedagogical principles on which educational inquiry is based and learning objectives, methods of instruction, learning style and pace of learning, assessment and evaluation strategies are connected with the critical reflection of reflective practitioners.

The above argument implies that teachers should develop strategies to motivate students to keep them focused as the instruction progresses and to consider that different students prefer different learning styles and that they learn at different rates. It also requires teachers to use appropriate technology to present and evaluate instruction as well as use relevant technology for follow-up learning activities to meet with students' interests and inspiring them to be self-regulated learners.

In a nutshell, technology in pedagogy is not a mere tool to provide students opportunities to learn what they want and needs, but guiding both teachers and students with thinking critically to enrich practical life experiences by developing the rationale to examine the appropriateness of the technological application. In a word, technology in pedagogy should not detach learners from a dynamic interaction and self-reflection to match learning objectives and show learners' autonomy. 